This item was submitted to Loughborough's Research Repository by the author.

Items in Figshare are protected by copyright, with all rights reserved, unless otherwise indicated.

\title{
Neurofunctional plasticity in fraction learning: An fMRI training study
}

PLEASE CITE THE PUBLISHED VERSION

https://doi.org/10.1016/j.tine.2020.100141

PUBLISHER

Elsevier

VERSION

AM (Accepted Manuscript)

PUBLISHER STATEMENT

This paper was accepted for publication in the journal Trends in Neuroscience and Education and the definitive published version is available at https://doi.org/10.1016/j.tine.2020.100141

\section{LICENCE}

CC BY-NC-ND 4.0

\section{REPOSITORY RECORD}

Wortha, SM, J Bloechle, M Ninaus, K Kiili, A Lindstedt, Julia Bahnmueller, Korbinian Moeller, and E Klein. 2020. "Neurofunctional Plasticity in Fraction Learning: An Fmri Training Study". Loughborough University. https://hdl.handle.net/2134/13109555.v1. 
Silke M. Wortha ${ }^{1,2}$, Johannes Bloechle ${ }^{3}$, Manuel Ninaus ${ }^{1,4}$, Kristian Kiili $^{5}$, Antero Lindstedt ${ }^{6}$, Julia

$$
\text { Bahnmueller }{ }^{1,7}, \text { Korbinian Moeller }{ }^{1,4,7,8} \& \text { Elise Klein }^{4,9}
$$

${ }^{1}$ LEAD Graduate School \& Research Network, University of Tuebingen, Tuebingen, Germany

${ }^{4}$ Leibniz-Institut für Wissensmedien, Tuebingen, Germany

${ }^{5}$ Faculty of Education and Culture, Tampere University, Tampere, Finland

${ }^{6}$ Faculty of Information Technology and Communication Sciences, Tampere University, Pori, Finland

${ }^{7}$ Centre for Mathematical Cognition, School of Science, Loughborough University, United Kingdom

${ }^{8}$ Individual Development and Adaptive Education Center, Frankfurt am Main, Germany

${ }^{9}$ Université de Paris, LaPsyDÉ, CNRS, Sorbonne Paris Cité, Paris, France

Corresponding author:

Silke M. Wortha

Department of Neurology, Universitätsmedizin Greifswald, Ferdinand-Sauerbruch-Straße, 17475 Greifswald Germany

silkemaria.wortha@med.uni-greifswald.de 
Abstract

39

Background: Fractions are known to be difficult for children and adults. Behavioral studies suggest that magnitude processing of fractions can be improved via number line estimation (NLE) trainings, but little is known about the neural correlates of fraction learning.

43 Method: To examine the neuro-cognitive foundations of fraction learning, behavioral performance and neural correlates were measured before and after a five-day NLE training.

Results: In all evaluation tasks behavioral performance increased after training. We observed a fronto-parietal network associated with number magnitude processing to be recruited in all tasks as indicated by a numerical distance effect. For symbolic fractions, the distance effect on intraparietal activation was only observed after training.

Conclusion: The absence of a distance effect of symbolic fractions before the training could indicate an initially less automatic access to their overall magnitude. NLE training facilitates processing of overall fraction magnitude as indicated by the distance effect in neural activation.

52

53 Keywords: fraction processing, number line estimation training, flow experience, numerical 54 distance effect, fMRI 55

Word count: 150

57 


\section{Introduction}

Over the last decade, research has shown repeatedly that understanding fractions is a crucial predictor of future achievement in higher mathematic [1-3]. However, despite intense research efforts in this area, children's poor performance when it comes to handling and understanding fractions hardly changed [4-7]. For example, in 2015 Lortie-Forgues and colleagues found that only $27 \%$ of $8^{\text {th }}$ graders in the United States were able to choose correctly the number closest to the result of a fraction addition problem out of four given solution probes [8]. A similar result was already reported 1978 by the National Assessment of Educational Progress [9], when only $24 \%$ of the $8^{\text {th }}$ graders chose the correct answer to the same question. Importantly, these difficulties in understanding fractions may be persisting regardless of educational efforts because - unlike natural numbers - fraction magnitude processing seems to be more difficult due to its bipartite structure reflecting the relative relation of numerator and denominator [10]. According to the integrated theory of numerical development, magnitude information is the crucial basis for understanding numbers. Moreover, the understanding that all real numbers (e.g., natural numbers, integers, rational numbers) can be represented on a number line is a key assumption for numerical learning. Therefore, promoting fraction magnitude understanding seems crucial for fostering fraction understanding more generally [11-13]. Thus, interventions with the aim to improve fraction understanding and therefore conceptual knowledge of fractions should focus on fostering mastery in processing and representing fraction magnitude. In the context of (fraction) magnitude understanding number lines have been shown to be a beneficial instructional tool [14].

Against this background, we aimed at understanding the neuro-cognitive foundations underlying successful fraction learning and their plasticity. For this purpose, participants had to complete a number line estimation training and a flow questionnaire on five consecutive days. In the following we will highlight the most important research results from the research areas which are relevant for our study. First, we will give a brief overview about the relevance of 
number line estimation training for fraction magnitude understanding. Second, we will introduce flow as a state which is beneficial for learning and especially for fraction learning. Third, we will summarize present knowledge about the neural correlates of fraction processing and highlight the importance of our study in this context. Finally, we will introduce the aim of the current study including our specific hypothesis.

\section{Number line estimation as predictor for fraction magnitude learning}

The mental number line is a metaphor for the nature of the number magnitude representation whereby numbers are represented spatially with their magnitude increasing from left to right (at least in Western cultures [15]). In numerical cognition research, number line estimation (henceforth NLE) is used repeatedly to assess number magnitude understanding especially in children ([16-18], but see [19] for additional aspects). In the NLE task, participants have to indicate the spatial position of a target number on a given number line for which only start- and endpoint are specified [18].

As magnitude is the semantic core for any type of number, the task can not only be used to assess, but also to train fraction magnitude understanding [12]. For instance, Hamdan \& Gunderson [20] conducted a training study with three conditions for fraction learning (i.e., number line estimation training, area model training, and a non-numerical control). They observed that although children in both the NLE training and the area model training improved in the respective tasks, only children completing the NLE training showed transfer effects to a not trained magnitude comparison task with fractions.

Moreover, Barbieri and colleagues [21] used a number line-based intervention to improve fraction understanding in children with poor conceptual knowledge of fractions and compared the number line intervention group to a standard mathematics curriculum group. The number line intervention group showed significantly larger learning gains than the control group. Finally, computerized and game-based versions of the NLE task where used successfully 
to assess and improve children's fraction understanding [22-24]. Taken together this substantiates that number lines are a powerful instructional tool and the NLE task can be applied successfully to foster fraction (magnitude) understanding. However, successful fraction magnitude learning might not only depend on improving conceptual knowledge of fractions, but also on a more fundamental ability to be able to reach a beneficial cognitive state for learning which is known as flow experience.

\section{Flow experience as an indicator for optimal learning}

Learning is not a pure cognitive process but is affected by motivation and emotions [25]. A beneficial factor for learning that is considered specifically in computerized approaches on learning is the flow experience of the learner [26]. Flow was first coined by Csikszentmihalyi [27] and can be described as a positive emotional state [28,29] and as a holistic approach to motivation [30]. In particular, flow is characterized by a combination of factors such as increased concentration, reduced self-consciousness, sense of control, that are experienced as intrinsically rewarding [29]. Flow is usually reached when task demands meet personal skills or resources in a balanced way. Thus, when the skills of the learner are too low for the demands of a given task - for instance at the beginning of a training - flow experience is rather low. The same is true when the skills of the learner are too high for a given task which leads to boredom and reduced flow experience. Therefore, flow experience seems to be an optimal state for intrinsically motivated learning, which helps focus on the given task and can lead to improved performance [31]. This is further supported by studies on the neural correlates of flow experience. These studies could show that flow experience is associated with increased activation in areas of the multiple demand system such as inferior frontal gyrus, putamen and anterior insula and decreased activation in areas typically associated with the default mode network such as amygdala, medial prefrontal cortex and posterior cingulate cortex $[32,33]$. 

learning settings. For instance, in game-based learning (for a review see [34]), collaborative learning of problem-solving in virtual environments [35], hypermedia learning [36], e-learning also be beneficial for fostering fraction magnitude understanding using a computerized NLE training. However, successful fraction magnitude learning might not only depend on improving conceptual knowledge of fractions and on the learners' flow experience, but also on the successful interplay of certain neural correlates underlying the neuro-cognitive foundations of fraction learning. Therefore, in the following the current state of research on neural correlates of fractional learning is briefly outlined.

\section{Neural correlates of fraction and proportion magnitude processing} research in educational sciences and psychology, little is still known about the neural mechanisms underlying the processing of proportions and fractions in general and the neural correlates of fraction learning in particular. To date, there are only few neuroimaging studies investigating the neural correlates of processing proportions [40-42] and fractions [43-46] in adults.

One important aspect across most studies is that the numerical distance effect was used as an indicator of automatic processing of overall fraction magnitude. The numerical distance effect [47] describes the finding that two numbers are compared faster and more accurately the larger the numerical distance between them (i.e., the farther apart they are on the mental number line, e.g. 3 and 7 is easier to compare than 3 and 4). For instance, for fraction magnitude comparison, Ischebeck et al. [45] observed that neural activation within the right IPS was modulated by the overall numerical distance between the to-be-compared fractions (e.g., 
numerical distance between $\frac{2}{4}$ and $\frac{3}{7}$ ), but not by the numerical distance between numerators or denominators (i.e., numerical distance between 2 and 3 for numerators and numerical distance between 4 and 7 for denominators when comparing $\frac{2}{4}$ and $\frac{2}{7}$ ). Moreover, Mock and colleagues [41] observed a joint neural correlate of specific occipito-parietal activation including the right intraparietal sulcus (IPS) for the processing of different notations of proportions including not only fractions, but also pie charts, dot patterns and decimals.

Finally, Klabunde et al. [48] conducted a first fMRI training study on proportions in participants with fragile $\mathrm{X}$ syndrome and a control group with intellectual disabilities. Participants were trained for two days in 10 min sessions until they were able to have over $80 \%$ accuracy on matching fractions to pie charts and pie charts to decimals. Neurofunctional changes from before to after the training indicated significantly increased brain activation in the left inferior parietal lobule, left postcentral gyrus, and left insula for both groups. However, the mechanism of interest in this study was not the distance effect but to investigate neural correlates of stimulus equivalence relations.

In summary, previous studies indicate that the distance effect for overall magnitude of the to-be-compared proportions/fractions seems a good measure reflecting automatic processing of overall fraction magnitude. As such, the numerical distance effect as a hallmark effect for magnitude processing indicated that the (right) IPS seems to play a crucial role in the processing of proportion and fraction magnitude independent of the actual task, which is in line with its involvement in the processing of natural number magnitude [49]. As such, one might argue that the presence of a numerical distance effect seems to indicate automatic processing of overall fraction magnitude in proficient fraction processing (see [50] for a similar argument on the relation between distance effect and arithmetic performance), while absence of a numerical distance effect might indicate that the fractions' magnitude is not automatically accessed. 


\section{The present study}

Against this background, we aimed at investigating neuro-functional correlates and their plasticity associated with an NLE training of fraction magnitude understanding. In particular, we evaluated changes in fraction magnitude processing on the neural level as reflected by the numerical distance effect for overall fraction magnitude. To the best of our knowledge, this is the first study investigating the neural correlates of fraction learning through a NLE training in healthy adult participants. We assessed neural activation associated with fraction magnitude processing using fMRI before and after a five-day consecutive NLE training on fractions. Additionally, we assessed participants' flow experience in each training session.

Similar to previous studies applying NLE training to children, we expected the training to improve participants' conceptual knowledge of fraction magnitude on a behavioral level [20,21]. In particular, we expected significant improvements in NLE performance over the fiveday training sessions. Additionally, we expected participants flow experience to be associated with NLE training improvements over the five-day training. Finally, on the neural level, we expected significant changes of IPS activation associated with the numerical distance effect from pre- to post fMRI session indicating more automatic processing of fractions' overall magnitude after the NLE training. This should become evident by a more pronounced numerical distance effect in behavioral measures but also neural activation in IPS after the training.

\section{Participants}

\section{Methods}

48 right-handed adult participants $(M=23.73, S D=3.65$, female $=32)$ took part in this fMRI study. All participants were German native speakers with normal or corrected to normal vision and reported no history of psychiatric or neurological disorders or drug abuse. The study was approved by the local Ethics Committee of the Medical Faculty of the University of Tübingen. Participants gave written informed consent and received monetary compensation for their participation. 


\section{Study Design}

The study was designed as a pre-post fMRI training study with five consecutive days of training between the fMRI measurements. On the first day before the training and on the last day after the training, participants had to complete two different magnitude comparison tasks (i.e., comparison of symbolic fractions and comparison of line proportions, respectively) and a fraction-line proportion matching task (i.e., indicating whether the magnitude reflected by a fraction matched that of a line proportion or not) while their brain activation was measured using fMRI (see Figure 1). In addition to these computerized tasks, participants also completed a paper-pencil-based NLE task prior to entering the scanner for pre- and post-test measurement (for more details see below). Due to technical problems, behavioral data of fMRI measurements could only be obtained from 24 right-handed adult participants $(M=22.50, S D=3.76$, female = 16). Imaging results did not differ substantially between the participant group with and without behavioral data (i.e., no supratheshold clusters at an uncorrected $p<.001$ and cluster size of 10). Therefore, imaging results as well as all behavioral data obtained outside the scanner (i.e., training data, flow experience and paper-pencil-based number line estimation task) will be participants only.

The training consisted of a fraction number line estimation task. It took place outside the scanner and each training session lasted around 15-20 minutes depending on participants individual performance. After each training session participants completed a brief questionnaire on mental flow to evaluate possible changes in flow experience over the training period (for more details on the flow questionnaire see below). 
number line estimation training $+$

flow short scale

Fig. 1: Study design. Before and after a five-day number line estimation training fMRI measurements were conducted to evaluate neurofunctional changes in brain activation through the training. After each training session flow was assessed using the flow short scale [51]. Prior to entering the scanner for the pre and post measurement a paper-pencil number line estimation task was administered.

In this study, two different item sets (one to be trained and one not to be trained) were

used. The order of sets was counterbalanced across participants so that whichever set a participant did not train on served as the untrained set at the pre- and post-test. However, each participant was tested during the fMRI sessions on both item sets. Each item set consisted of 48 stimuli with items always presented in randomized order. To ensure comparable difficulty of item sets, they were matched on overall problem size and numerical distance between and denominators ranging from 1 to 30 . To evaluate neurofunctional changes in fraction magnitude processing, pre-post-test evaluation tasks performed in the fMRI scanner included both trained and untrained items. Items of the two item sets can be found in the Appendix. Training Procedure training with feedback, which differed in appearance and framing, but not in numerical content and task. In particular, half of the participants were trained using an NLE task set within a gamified environment (see Figure 2), while the other half were trained in the same NLE task in 
research engine, which was already applied successfully in previous studies for assessing and training fraction knowledge [23,24,52-54].

In both versions of the NLE task, participants had to indicate the correct position of a given fraction on a number line ranging from 0 to 1 by maneuvering an avatar in the gamified version and moving a white slider along the number line using the left and right arrow keys of a computer keyboard in the non-game version. After reaching the estimated correct position, participants had to press the space bar to select that position. Participants were instructed to indicate the right position as quickly and accurately as possible within a time limit of 10 seconds. They received positive feedback (i.e., cheering avatar plus coins awarded in the gamified vs. green checkmark in the non-game version) when their answer fell within a range $\pm 5 \%$ around the correct position. In case they failed to answer or did not answer accurately enough, negative feedback was given (i.e., avatar struck by lightning plus loss of virtual energy in the gamified vs. red cross shown in the non-game version) and participants had to repeat the item until it was correctly solved within the $\pm 5 \%$ range. At each training session, participants worked through 96 items in 12 runs containing 8 items each. Each item from the trained stimulus set was encountered twice within a training session. Items were presented in randomized order and were identical in both versions of the NLE training.

I. Gamified Version

A

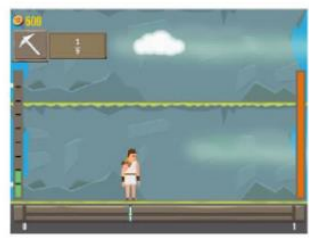

C

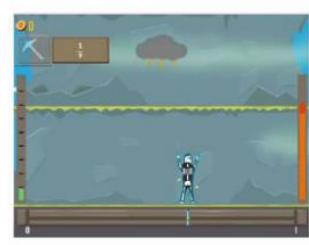

B

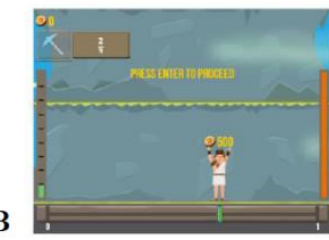

D

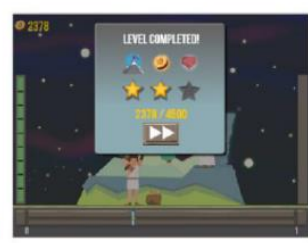

II. Non-Game Version

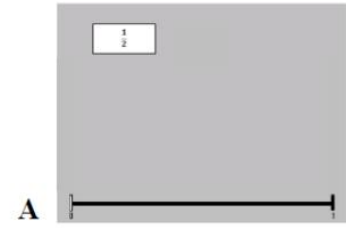

B

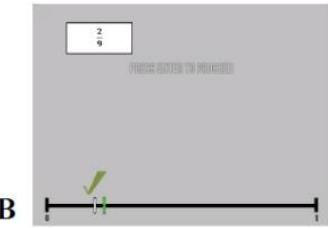

$\square$

D

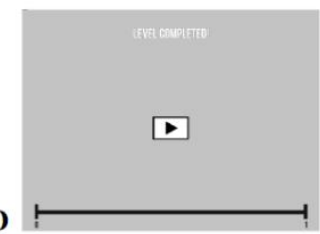




\section{Flow Short Scale}

questionnaire consists of 16 items. Thirteen items are associated with the flow scale (7-point

Likert-scale ranging from $1=$ totally disagree to $7=$ totally agree), which has a three-

items, i.e., "My thoughts or activities run fluently and smoothly", $\alpha=.92$ ). The second

right now", $\alpha=.80$ ). Finally, the third dimension is perceived importance or concern (3 items,

i.e., "I'm worried about failure", $\alpha=.90)$.

Additionally, the questionnaire includes 3 items of a demand scale (10-point Likertscale ranging from $1=$ easy to $10=$ difficult), which aims at assessing how demanding the

current task was for the participant (i.e., "For me personally, the current requirements are....").

To monitor flow experience over the course of the training, participants had to complete the

flow short scale following each completed training session resulting in five completed flow

short scales per participant.

\section{Paper pencil number line estimation task outside the fMRI scanner}

In addition to the computerized in-scanner tasks (see below) participants had to complete a paper pencil version of a number line estimation task prior to both fMRI 
only the endpoints specified and participants had to indicate the spatial position on the number line for all items from both item sets (i.e., the trained and the untrained set, thus 96 items in total prior to and after the training). This task allowed to evaluate potential improvements in spatial localization of fractions on the number line through the training.

\section{Tasks performed inside the fMRI scanner}

For the fMRI paradigm a block design was used, with four separate blocks for the three different tasks (i.e., fraction-line proportion matching task, line proportion comparison task and symbolic fraction magnitude comparison task). For each item in the fraction-line proportion matching task we presented a matching (i.e., magnitude of symbolic fraction and line proportion were identical) and a non-matching version (i.e., magnitude of symbolic fraction and line proportion were not identical) in the fraction-line proportion matching task. Therefore, this task took twice as long than both comparison tasks and was run in two blocks.

In the fraction-line proportion matching task, participants were shown a fraction and a line proportion (see Figure 3a). They had to indicate whether the fraction and the line proportion reflected the same magnitude or not (i.e., identical: left button, different: right button). In half of the items, magnitudes of fraction and line proportion matched. In the line proportion comparison task (see Figure 3b), participants were shown two line proportions and had to decide which proportion was the numerically larger one by pressing a corresponding response button (i.e., the right button when the upper and the left button when the lower line proportion was larger). Similar, in the symbolic fraction magnitude comparison task (see Figure 3c), participants were shown two fractions and had to decide which fraction was numerically larger again.

Each block consisted of 4 practice followed by 48 critical trials. In both sessions, half of the critical trials consisted of trained stimuli while the other half were untrained stimuli. Stimulus order was random for each participant and each session. Additionally, 22 trails of a 
scrambled word task were randomly interspersed in each block of each condition to control for

eye-movements and to control that participants would stay focused. During these trials, two strings of scrambled letters were shown on top of each other and participants had to decide which of the strings would form a real word (see Figure 3d). $=255255255)$ were randomly interspersed between trials. All stimuli were projected on a screen outside the scanner and made visible to participants through a mirror mounted on the head coil of the scanner. Foam pads were used to minimize head movements within the head coil during fMRI acquisition. Stimuli were presented in black font against a white background $\left(\right.$ RGB values $=255255$ 255). The experiment was programmed using Presentation ${ }^{\circledR}$ v16.1 software (www.neurobs.com).
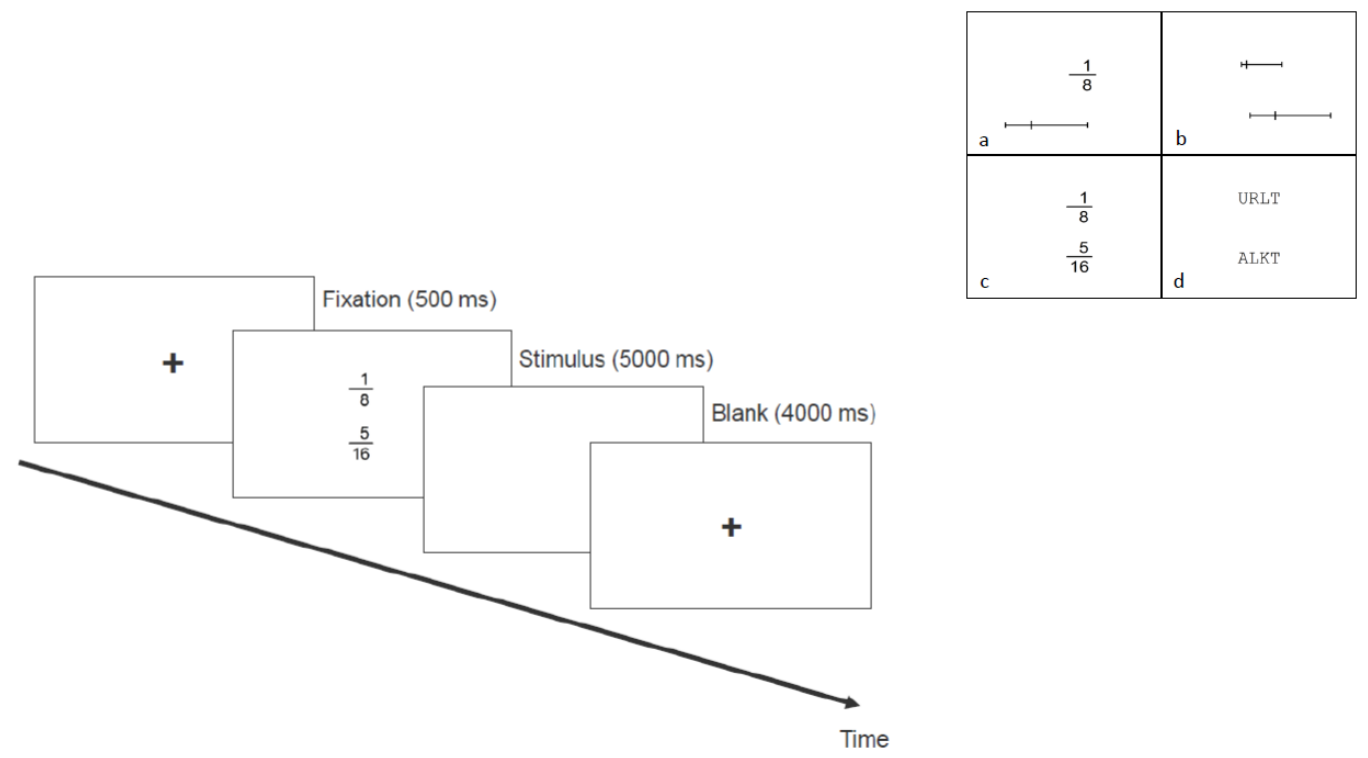

Fig. 3: Experimental procedure with examples (upper right box) for the different tasks. Example for a) the fraction-line proportion matching task, b) the line proportion comparison task, c) the symbolic fraction magnitude comparison task, and d) the scrambled letters control task. In this example the lower four letters can be unscrambled to form the word "kalt" (German word for "cold"). The upper four letters cannot be formed to any word used in German. 

which appeared for $5000 \mathrm{~ms}$ or until participants responded. Subsequently, a blank screen appeared for $4000 \mathrm{~ms}$ followed by the beginning of a new trial (see Figure 3). Participants responded by pressing one of two MRI compatible response buttons with either their left or their right thumb. Participants were instructed to answer as fast and as accurately as possible.

\section{MRI and fMRI Acquisition}

A high-resolution T1-weighted anatomical scan was acquired by a $3 \mathrm{~T}$ Siemens Magnetom Prisma MRI system (Siemens AG; Erlangen, Germany) equipped with a 64-channel head-neck matrix coil $(\mathrm{TR}=2400 \mathrm{~s}$, matrix $=256 \times 256,176$ slices, voxel size $=1.0 \times 1.0 \times$ $1.0 \mathrm{~mm}^{3} ; \mathrm{FOV}=256 \mathrm{~mm}, \mathrm{TE}=2.92 \mathrm{~ms}$; flip angle $=8^{\circ}$ ). The anatomical scan was always performed at the end of each experimental session.

Functional $\mathrm{T} 2 *$-weighted images were obtained using multiband gradient-echo Echo planar imaging $\left(\mathrm{EPI} ; \mathrm{TR}=792 \mathrm{~ms} ; \mathrm{TE}=30 \mathrm{~ms} ;\right.$ flip angle $=58^{\circ} ; \mathrm{FOV}=192 \mathrm{~mm}, 64 \times 64$ matrix; 48 slices, voxel size $=3.0 \times 3.0 \times 3.0 \mathrm{~mm}^{3}$ ). Total scanning time was approximately 80 minutes. A baseline (rest) condition was accomplished by including about $20 \%$ null events in the paradigm.

\section{Analysis}

Preliminary Analysis

Prior to the analysis of the behavioral and imaging data of the present study, possible differences between the two variants of NLE training (gamified vs. non game-based training) were examined both on the behavioral as well as the neuro-functional level. However, the behavioral analysis after the training showed no significant differences in reaction times or error rates for all three evaluation tasks performed in the scanner (i.e., symbolic fraction magnitude comparison, line proportion comparison, and fraction-line proportion matching task, all $p$ - 
values $>.05$, all $F s \leq 2.85)$. In line with this, the analysis of imaging data revealed no significant difference between the two groups after the training for any of the three evaluation tasks at an uncorrected $p$-value of .001 with a cluster size of $k=10$ voxels. Because neither behavioral nor neurofunctional differences were observed for the two trainings, we decided to merge both training groups in order to investigate fraction learning across groups with higher statistical power.

Behavioral analysis

\section{Training and flow data}

To evaluate learning outcomes over the five training time points and associations with participants' flow experience during learning we used a latent growth linear mixed-effects model over the five training time points. Regarding dependent variables, we were interested in the mean percentage absolute estimation error (PAE; [55]) and the number of attempts participants needed to estimate a given fraction correctly. Models were fitted in R using 'lmer' from the 'lme4' package [56]. To provide $p$-values we used the 'summary' function of the "ImerTest" R package [57]. Summary statistics were extracted via the 'analyze' function of 'psycho' [58].

\section{Number line estimation task}

Mean PAE (cf. [55]) was calculated to reflect performance in the number line estimation task at the two time points. Items without a response were not considered for analyses. To evaluate performance changes in PAE between the pre- vs. post-test, a linear mixed-effects model was fitted using 'Imer' from the "Ime4" R package [56]. Again, to provide $p$-values we used the "summary' function of the "ImerTest" R package [57]. Additionally, summary statistics were also extracted via the 'analyze' function of "psycho” R package [58]. 


\section{Behavioral fMRI data}

For the analysis of the behavioral fMRI data we evaluated reaction times and accuracy as dependent variables. Three separate linear mixed-effects models were run to analyze reaction times for the three different evaluation tasks. Items without a response and items answered incorrectly were not considered for reaction time analyses. To analyze error rates and to include participants' individual performance as random effect, we ran three separate generalized linear mixed-effects models (GLMM) fitted by using the R package 'Ime4' [56]. In the GLMMs we assumed a binomial error distribution and used the logit as the link function. For both types of analysis, we provided $p$-values with the 'summary' function of the "ImerTest" R package [57].

Imaging analysis

fMRI data analyses were performed using SPM12 (http://www.fil.ion.ucl.ac.uk/spm). Images were motion corrected and realigned to each participant's mean image. The mean image was co-registered with the anatomical whole-brain volume. Imaging data was then normalized into standard stereotaxic MNI space (Montreal Neurological Institute, McGill University, Montreal, Canada). Images were resampled every $2.5 \mathrm{~mm}$ using 4th degree spline interpolation to obtain isovoxels and then smoothed with a $5 \mathrm{~mm}$ full-width at half-maximum (FWHM) Gaussian kernel to accommodate inter-subject variation in brain anatomy and to increase signalto-noise ratio in the images. Data were high-pass filtered (128s) to remove low-frequency noise components and corrected for autocorrelation assuming an $\mathrm{AR}(1)$ process. Brain activity was convolved over all experimental trials with the canonical hemodynamic response function (HRF) and its first time derivative.

For the first level analysis, pre- and post-fMRI training sessions were combined on the subject level in a generalized linear model (GLM). For each participant, we considered the two factors item-set (trained vs. untrained) and session (pre vs post). This resulted in four experimental conditions: trained pre (T1), trained post (T2), untrained pre (UT1) and untrained 
post (UT2). Additionally, to evaluate the influence of fraction problem difficulty we included the covariate numerical distance between to-be-compared fractions as a parametric regressor in the first level analysis. As a control variable the scrambled word problems (hereafter revered to as "words") were also included in the first level. Finally, the six movement parameters from preprocessing were entered into the model to capture signal variations due to head motion.

These images then entered the second level random-effects group analysis using a flexible factorial design. The SPM Anatomy Toolbox [59], available for all published cytoarchitectonic maps (http://www.fz-juelich.de/ime/spm_anatomy_toolbox), was used for anatomical localization of effects where applicable. For areas not yet implemented, the anatomical automatic labelling tool (AAL) in SPM12 (http://www.cyceron.fr/web/aal anatomical_automatic_labeling.html) was used.

All contrasts calculated reflect the parametric modulation of the fMRI signal by the numerical distance between two proportions presented (distance effect). Simple contrasts (distance effect in each notation) were family-wise error corrected at $p<.05$ at the whole-brain level with a cluster size of $k=10$ voxels. Complex contrasts comparing two distance effects (e.g., distance effect in fractions after training vs. distance effect prior to the training) were thresholded at an uncorrected $p$-value of $<.001$ at the voxel level with a cluster size of $k=10$ voxels and were reported when they remained significant following family-wise error correction (FWE) at the cluster-level with $p_{\text {cluster-corr }}<.05$.

Behavioral Results

\section{Training and Flow Experience}

Percentage absolute estimation error

Differences in PAE and possible associations with flow experience over time were analyzed using a latent growth linear mixed effect model, predicting PAE by flow experience and time (i.e., five training time points) as fixed factors while also including a random intercept 
to account for participants' individual differences in prior knowledge. The model explained a significant proportion of variance in PAE $\left(R^{2}=75.22 \%\right.$; fixed effects: $\left.R^{2}=12.97 \%\right)$ and showed that $\mathrm{PAE}[\beta=-0.34, \mathrm{SE}=0.15, t(193)=-2.29, p<.05]$ significantly improved over time (see Figure 4A for PAE changes over time). Moreover, the fixed effect of flow $[\beta=-0.02, \mathrm{SE}=$ $0.01, t(198)=-3.13, p<.01]$ was significant, indicating that flow experience changed over time (see Figure 4C for general changes in flow experience over time). The interaction between time and flow experience was not significant $[t(193)=0.62, p=.54]$.

\section{Number of attempts}

Identical to the first analysis, differences in the number of attempts participants needed to estimate the given fraction during the NLE training correctly over time and possible associations with flow experience were analyzed using again a latent growth linear mixed effect model. Number of attempts needed was predicted by participants' flow experience and time (i.e., five training time points) as fixed factors. Again, we included a random intercept to account for participants individual differences in prior knowledge. The model explained a significant proportion of variance in the number of attempts needed to estimate the given fraction correctly $\left(R^{2}=71.24 \%\right.$; fixed effects: $\left.R^{2}=16.16 \%\right)$ and showed that the number of attempts significantly decreased over time $[\beta=-0.18, \mathrm{SE}=0.07, t(194)=-2.70, p<.01$; see Figure 4B for number of attempts changes over time]. Within this model the fixed effect of flow was significant $[\beta=-0.01, \mathrm{SE}=0.06, t(201)=-3.40, p<.001]$, indicating that flow experience changed over time (see Figure 4C for general changes in flow experience over time). Again, the interaction between time and flow experience was not significant $[t(194)=0.95, p=.34]$. 

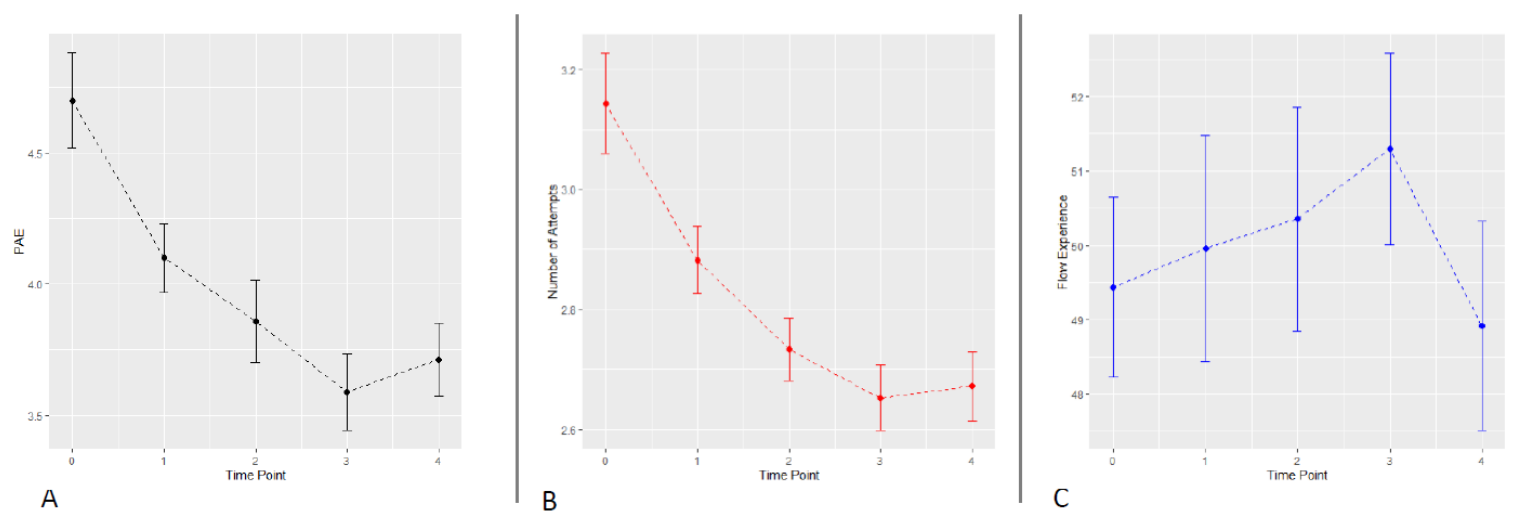

Fig. 4: Improvement of PAE (A), number of attempts needed (B), and changes in flow experience (C) over the training period (i.e., five time points). Error bars indicate standard errors (SEM).

\section{Number line estimation task}

To investigate whether PAE for the paper pencil-based NLE tasks differed between preand post-test we ran another linear mixed effect model. We defined the two time points (pre vs. post) as fixed effect and included a random intercept for subjects to account for individual variability. The overall model predicting differences in PAE on the two number line estimation tests (pre vs. post) explained a significant proportion of variance $\left(R^{2}=63.34 \%\right.$, in which the fixed effects explained $R^{2}=5.56 \%$ of the variance). The effect of session was significant $[\beta=$ $-0.01, \mathrm{SE}=0.00, t(47)=-3.80, p<.001]$ indicating that performance was better on the posttest than the pretest (reflected by smaller estimation errors).

\section{Behavioral fMRI results}

\section{Fraction-line proportion matching task}

Performance changes in reaction time between pre- and post fMRI sessions for the fraction-line proportion matching task were evaluated by a linear mixed effect model. We defined session (pre vs. post), itemset used (trained vs. untrained), and numerical distance (i.e., only for the non-matching items) as fixed effects. Moreover, interactions between session and item set as well as session and numerical distance were also included as fixed effects to evaluate 
whether the possible influence of item set or numerical distance on participants reaction time changed from pre- to post-test. Finally, we included a random intercept to account for participants individual differences in prior knowledge.

The model explained significant proportions of variance on participants reaction times $\left(R^{2}=26.18 \%\right.$; fixed effects: $\left.R^{2}=9.74 \%\right)$ and showed that reaction times significantly improved from pre- to post-test $[\beta=-260.85, \mathrm{SE}=89.37, t(1068)=-2.92, p<.01]$. Additionally, the fixed effect of numerical distance was significant $[\beta=-1306.84, \mathrm{SE}=210.59, t(1069)=-6.21$, $p<.001]$, indicating that reaction times improved more strongly for increasing distances. Neither the fixed effect of itemset nor the interactions were significant [all $t \leq 0.48$, all $p_{\mathrm{s}}>.05$ ]

For the evaluation of performance changes in accuracy between pre- and post-test, we ran a generalized linear mixed-effects model, by using logit as the link function and assuming a binomial distribution of the error rates. To avoid overfitting of the model we only included session, itemset used and numerical distance as fixed effects and a random intercept accounting for individual differences in prior knowledge. The model revealed a significant fixed effect of numerical distance $[\mathrm{z}=-6.86, p<.001]$, indicating that increasing distances between fractions and line proportions led to less errors. The fixed effects of session and trained item were not significant [all $\mathrm{z} \leq-0.18$, all $\left.p_{\mathrm{s}}>.05\right]$.

\section{Line proportion comparison task}

Identical to the analysis of the fraction-line proportion matching task we ran the same linear mixed-effects model (including session, itemset used, numerical distance, as well as the interaction session and item set and the interaction session and numerical distance as fixed effects and a random intercept to account for individual differences in prior knowledge) to investigate possible performance changes in reaction time on the line proportion comparison task between pre- and post fMRI session. The model explained a significant proportion of variance on participants reaction times $\left(R^{2}=42.71 \%\right.$; fixed effects: $\left.R^{2}=8.57 \%\right)$ and showed 
that reaction times significantly improved from pre to post-test $[\beta=-279.33, \mathrm{SE}=118.19$, $t(507)=-2.36, p<.05]$. Additionally, the fixed effect of numerical distance was significant $[\beta$ $=-1686.98, \mathrm{SE}=295.05, t(510)=-5.72, p<.001]$, indicating that reaction times got significantly faster with increasing distances. Neither the fixed effect of itemset nor the interactions were significant [all $\mathrm{t} \leq 0.35$, all $\left.p_{\mathrm{s}}>.05\right]$.

Again, to investigate possible performance changes in accuracy for the line proportion comparison task between pre- and post-test, we ran a generalized linear mixed-effects model, using logit as the link function and assumed a binomial distribution of the error rates. To avoid overfitting of the model we only included session, itemset used and numerical distance as fixed effects and a random intercept accounting for individual differences in prior knowledge. Analyzing error rates for the line proportion comparison task revealed a significant fixed effect of numerical distance $[\mathrm{z}=-7.5, p<.001]$, indicating that increasing distances between line proportions led to less errors. However, there were no significant differences for session and item set [all $\mathrm{z} \leq-1.10$, all $\left.p_{\mathrm{s}}>.05\right]$.

\section{Symbolic fraction magnitude comparison task}

Finally, possible performance changes in reaction time for the symbolic fraction magnitude comparison task between pre- and post-test were tested identical to the prior two evaluation tasks. We again ran a linear mixed-effects model with session, itemset used, numerical distance as well as the interaction session and item set and the interaction session and numerical distance as fixed effects and a random intercept accounting for individual differences in prior knowledge. Interestingly, in this model only the fixed effect for numerical distance was significant $[\beta=-1282.76, \mathrm{SE}=337.30, t(499)=-3.80, p<.001]$, indicating that reaction times were significantly faster for increasing distances. Neither the fixed effects of session and itemset nor the interactions were significant [all $\mathrm{t} \leq 0.43$, all $\left.p_{\mathrm{s}}>.05\right]$. 

comparison task between pre- and post-test, were evaluated again by a generalized linear mixed-effects model, using logit as the link function and assuming a binomial distribution of the error rates. To avoid overfitting of the model we only included session, itemset used and numerical distance as fixed effects and a random intercept accounting for individual differences in prior knowledge. Thus, analyzing error rates for the fraction task revealed a significant fixed effect of session $[\mathrm{z}=-2.38, p<.05]$, indicating that participants performed better in the posttest compared to the pre-test. Additionally, the fixed effect of numerical distance was significant $[\mathrm{z}=-9.17, p<.001]$, indicating that increasing distances between fractions led to less errors when comparing two fractions. However, there was no significant difference for item set $[\mathrm{z}=$ $0.282, p=.079]$.

\section{Imaging results}

Distance effect before the training

\section{Fraction-line proportion matching task}

Processing of smaller numerical distances between fractions and line proportions in the fraction-line proportion matching task was associated with stronger magnitude-specific fMRI signal before the training in a bilateral fronto-parietal neural network including areas in the intraparietal cortex (hIP3), the superior parietal cortex (SPL), the inferior frontal gyrus (Areas 44 and 45), bilateral inferior temporal gyri as well as bilateral insula. Further activated clusters were found in the bilateral middle frontal gyri as well as right-hemispheric subcortical areas such as thalamus and caudate nucleus as well as the cerebellum (see Table 1, Figure 5A and C depicted in red color).

\section{Line proportion comparison task}



modulated the fMRI signal before the training in a right-hemispheric fronto-parietal network centered around the right intraparietal sulcus (hIP3). Smaller numerical distance between proportions led to stronger activation in the right IPS and the right anterior IPS reflecting

\section{Symbolic fraction magnitude comparison task}

Activation in no cluster of voxels was modulated significantly by numerical distance at the given threshold in the symbolic fraction magnitude comparison task before the training.

Table 1: Distance effect for line proportion comparison and fraction-line proportion matching task at a familywise error corrected $p<.05$, cluster size $k=10$ at the whole brain level.

\begin{tabular}{llrrrrr}
\hline \multirow{2}{*}{ Contrast } & Brain region & MNI (x, y, z) & $\begin{array}{c}\text { Cluster } \\
\text { size }\end{array}$ & $t$ \\
\hline \multirow{2}{*}{ Distance effect } & RH anterior intraparietal sulcus (hIP2) & 43 & -36 & 47 & 61 & 5.63 \\
Line proportion & RH intraparietal sulcus (hIP3) & 33 & -52 & 53 & 12 & 5.04 \\
& RH inferior frontal gyrus (44) & 51 & 8 & 23 & 15 & 5.61 \\
& & & & & & \\
Distance effect & RH precentral gyrus & 38 & -20 & 55 & 2195 & 11.49 \\
Matching task & RH intraparietal sulcus (hIP3) & 26 & -57 & 55 & & 7.15 \\
& RH superior parietal lobe (SPL) & 18 & -60 & 58 & & 5.46 \\
& LH intraparietal sulcus (hIP3) & -30 & -58 & 42 & 531 & 7.29 \\
\hline
\end{tabular}




\begin{tabular}{lrrrrr}
\hline LH superior parietal lobe (SPL) & -25 & -57 & 53 & & 7.01 \\
LH inferior frontal gyrus (IFG 44) & -42 & 5 & 30 & 167 & 7.71 \\
RH inferior frontal gyrus (IFG 44) & 53 & 8 & 28 & 179 & 7.38 \\
LH inferior frontal gyrus (IFG 45) & -40 & 28 & 23 & 85 & 6.77 \\
LH middle frontal gyrus & -20 & 6 & 55 & 58 & 6.25 \\
RH middle frontal gyrus & 41 & 41 & 18 & 85 & 5.91 \\
LH posterior medial frontal gyrus & -7 & 8 & 58 & 271 & 7.24 \\
RH inferior temporal gyrus & 51 & -52 & -10 & 22 & 5.91 \\
LH inferior temporal gyrus & -50 & -57 & -10 & 30 & 5.79 \\
RH thalamus & 18 & -22 & 10 & 68 & 7.57 \\
RH caudate nucleus & 8 & 16 & 3 & 20 & 6.24 \\
RH insula & 33 & -20 & 15 & 86 & 6.76 \\
RH insula & 31 & 28 & 3 & 54 & 6.08 \\
RH insula & 41 & 1 & 10 & 13 & 5.75 \\
LH insula & -35 & 18 & 3 & 17 & 5.55 \\
LH cerebellum & -17 & -52 & -23 & 306 & 10.56
\end{tabular}

A

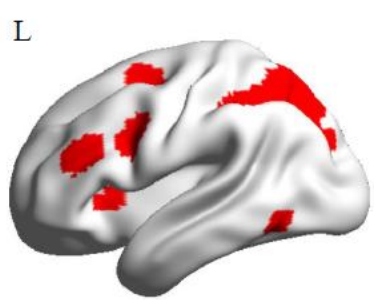

B

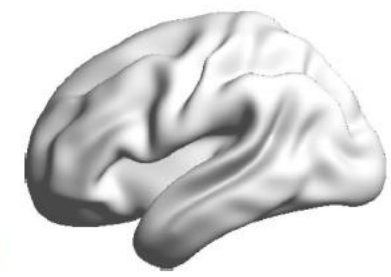

C

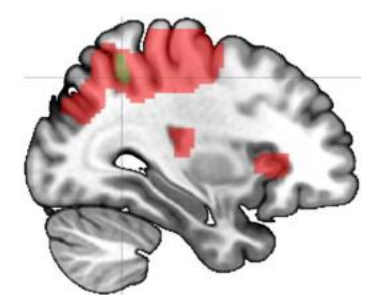

604
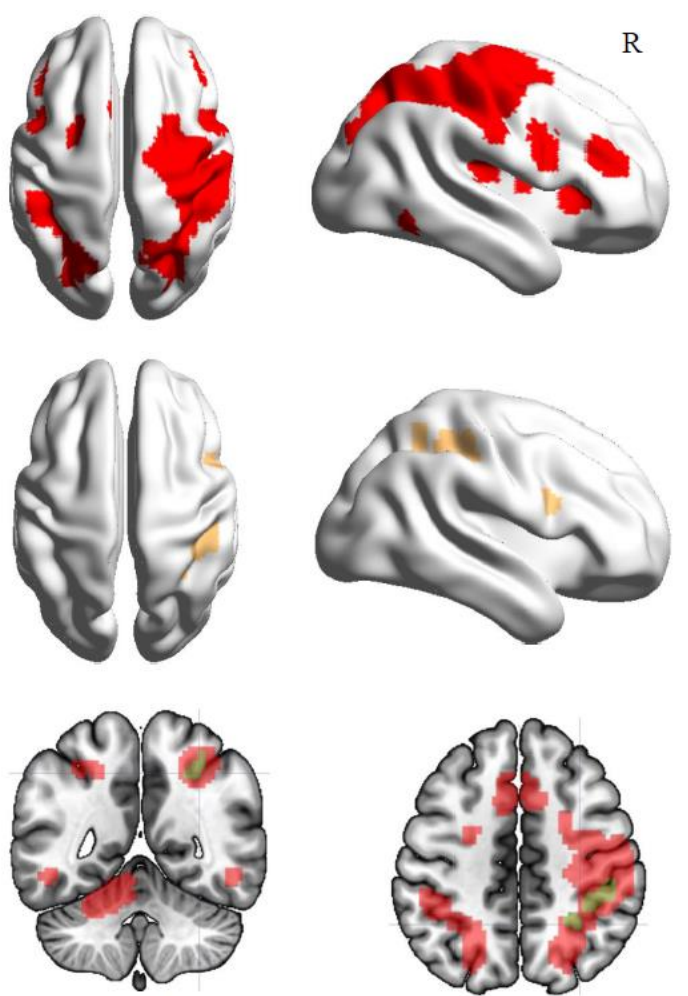

$y=-51$

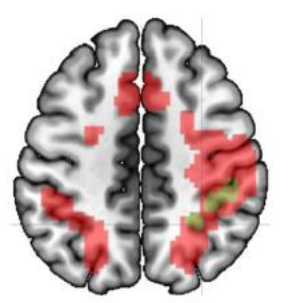

$z=45$ 
Figure 5: Effect of overall magnitude processing in the fractions-lines matching task (Panels A and C: red) and the lines proportion comparison task (Panels B and C: gold) as reflected by the distance effect.

Training and transfer effects

Line proportion comparison / fraction-line proportion matching task

When comparing the distance effect after the training to the distance effect before the training, no suprathreshold clusters of activation were observed for the line proportion comparison or in the fraction-line proportion matching task.

\section{Symbolic fraction magnitude comparison task}

Direct comparison of the distance effect after the training to the distance effect before the training in the symbolic fraction magnitude comparison task revealed significant increased activation differences in a bilateral fronto-parietal network centered around the intraparietal sulcus (hIP3; see Table 2, Figure 6). Further clusters of significant increased activation differences were observed in the right superior parietal lobe (SPL) and the left inferior parietal lobe $(\mathrm{PFt})$, the right fusiform gyrus, the bilateral frontal cortex and the left thalamus.

When comparing distance effects observed for the trained item set to those observed for untrained item set after the training, no suprathreshold clusters of activation were observed, indicating that the training effect was comparably strong for trained as well as untrained fraction items. This means that for fraction magnitude processing it seemed that the training effect generalized to untrained items after the NLE training.

Table 2: Effect of training for processing of overall fraction magnitude as reflected by the distance effect for the symbolic fraction magnitude comparison task.

\section{Contrast}




$\begin{array}{llrrrrr}\text { Fractions post vs. } & \text { RH superior parietal lobe (SPL) } & 26 & -45 & 45 & 59 & 4.42 \\ \text { pre training } & \text { RH intraparietal sulcus (hIP3) } & 31 & -60 & 48 & 21 & 3.70 \\ & \text { LH intraparietal sulcus (hIP3) } & -27 & -62 & 50 & 15 & 3.53 \\ & \text { LH supramarginal gyrus (PFt) } & -34 & -28 & 33 & 55 & 4.56 \\ & \text { LH middle frontal gyrus } & 26 & 1 & 40 & 80 & 5.36 \\ & \text { RH posterior medial frontal gyrus } & 13 & 11 & 53 & 133 & 4.08 \\ & \text { RH inferior frontal gyrus (IFG) } & 41 & 36 & 15 & 45 & 4.05 \\ & \text { RH fusiform gyrus } & 36 & -47 & -18 & 15 & 3.51 \\ & \text { LH thalamus } & -17 & -5 & 3 & 18 & 5.61 \\ & \text { LH cuneus } & -25 & -57 & 28 & 15 & 4.07\end{array}$
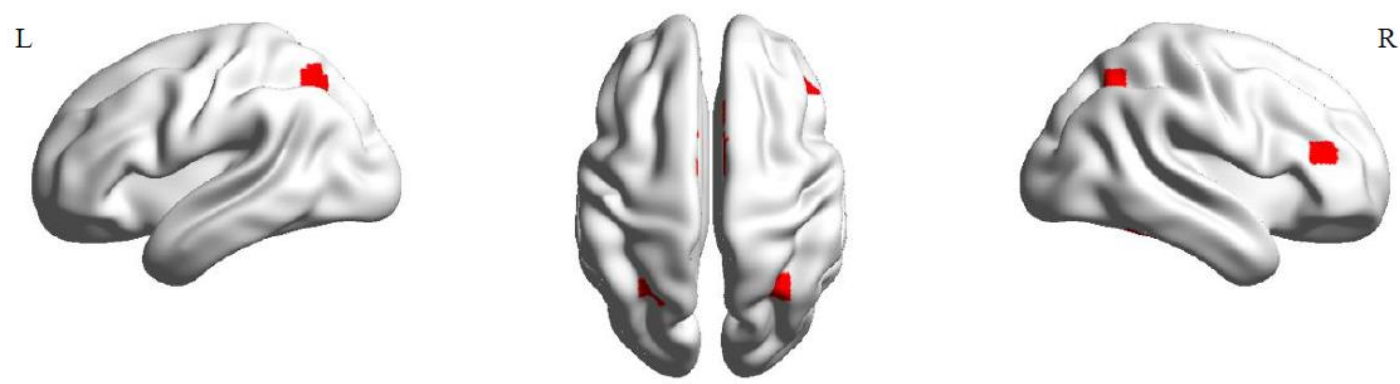

$\mathrm{R}$

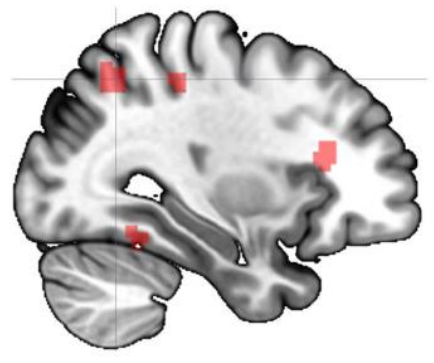

$\mathrm{x}=32$

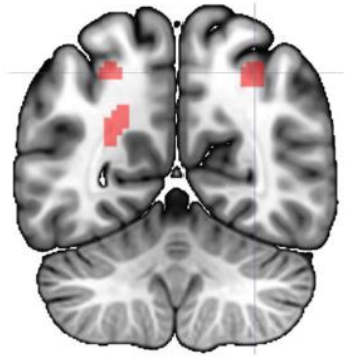

$y=-58$

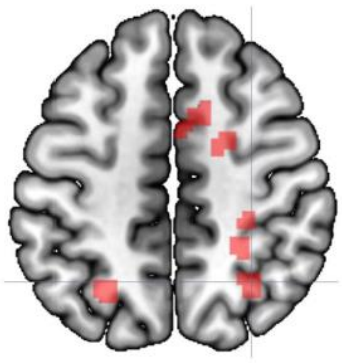

$z=48$ 
The aim of the present study was to investigate potential neuro-functional changes of brain activation in adult participants through a five-day consecutive NLE training on fraction magnitude. While there already exist a number of studies indicating the effectiveness of NLE training on the behavioral level $[20-22,60]$ and some few studies investigating the neural correlates of fraction and proportion processing [41,42,44,45], little is still known about the neural correlates of fraction learning.

Evaluation tasks included symbolic fraction and non-symbolic line proportion magnitude comparison tasks and a fraction-line proportion matching task. Additionally, learning trajectories over the five time points of the NLE training, participants' corresponding flow experience as well as a paper pencil pre- post NLE task were evaluated. In the following, behavioral as well as neurofunctional results for these measures will be discussed in more detail.

\section{The interplay between training performance and flow experience}

Flow experience has been described as an optimal state for intrinsically motivated learning, which helps focus on the given task and can lead to improved performance [31]. Another explanation for optimal learning was first described by Yerkes and Dodson in 1908 and refers to the relationship between arousal and performance (i.e., Yerkes-Dodson-Law; [61]). This law states that learners' optimal performance is achieved on a medium level of arousal reflected by an inverted U-shape relation between the respective parameters. Transferred to flow experience this inverted U-shape suggests that a balance between cognitive demands of the task at hand and individual skill level is the basis for best possible flow experience. Thus, when skills of a learner are too poor for the demands of a given task - for instance at the beginning of a training - flow experience may be rather low. The same is true when skills of a learner are too advanced for a given task. Both non-optimal states can lead to boredom and/or frustration, and reduced flow experience. In turn, this might interfere with learning of the given task [62]. 
In line with these assumptions, our results for training performance and flow experience

over the five day NLE training indicated that at the beginning of the training participants' flow experience was significantly lower and PAE as well as number of attempts needed to solve a trial successfully were significantly higher as compared to later training days. This possibly reflects an imbalance between task demands and individual skills. PAE and number of attempts needed on the first training day suggest that participants' ability on the task seemed to be low in the beginning. With each training day passing flow experience increased while at the same time PAE and number of attempts needed decreased. Thus, participants experienced a more and more optimal learning situation in which demands of the task and individual skill level were in balance.

Interestingly, on the last training day participants' flow experience dramatically decreased again to values lower than on the first training day. This was accompanied by slight increases of PAE and number of attempts needed. Thus, we assume that participants peak of performance was already achieved at the $4^{\text {th }}$ training day. We can only speculate whether this was caused either by boredom or the fact that fraction magnitudes could not be estimated more accurately by our participants after 4 days of training. Importantly, however, this decrease in flow experience was not associated with a general decrease in training performance. Moreover, we observed that participants significantly improved in the paper pencil NLE task from pre to post session.

\section{Transfer effects in fraction and proportion learning}

Behavioral data indicated significant performance improvements for all three tasks. Importantly, these improvements did not differ between trained and untrained items, indicating transfer effects of the training to untrained items. Additionally, neurofunctional data showed similar results: before the training no significant differences in brain activation were observed between the two stimulus sets (trained and untrained set) for all three evaluation tasks. After the training, again, no suprathreshold clusters of activation were observed when comparing 
trained and untrained items for all three evaluation tasks. Thus, indicating that the training effect was comparably strong for trained as well as untrained items and seemed to generalize to untrained items after the NLE training.

Moreover, as discussed in more detail below, for the case of the fraction magnitude comparison task we think brain activation associated with numerical distance after the NLE training indicates that overall symbolic fraction magnitude was not automatically processed before training.

However, one might argue that our results were elicited by the applied drill-like training approach. In the literature, this is often used to investigate numerical learning in terms of arithmetic fact learning $[63,64]$. Nevertheless, we are confident that participants did not just learn specific fraction magnitudes by heart for at least two reasons: First, if fraction magnitudes were learned by drill no transfer effect from trained to untrained items should be evident neither on the behavioral nor on the neural level. Moreover, fractions that are learned by heart should not show a numerical distance effect especially for untrained items because their overall magnitude should not be processed. In our study induced automated magnitude activation was also present for untrained items. Thus, it is unlikely that our training supported fact learning but rather improved magnitude representation.

Second, previous literature reported different neural correlates for arithmetic fact learning than observed in the current study. In particular, learning arithmetic facts is associated with a shift from bilateral fronto-parietal processing around the IPS to a primarily left hemispheric network in the medial temporary lobe (MTL) involving the hippocampus (cf. [6466]). However, in the present study, we rather observed a shift towards more activation within the fronto-parietal network of magnitude processing [67-69] - thus, indicating more explicit processing of overall fraction magnitude and not fact retrieval after the training. 
This supports the notion that the training indeed resulted in a general conceptual improvement and automatization of fraction magnitude processing, in contrast to training fact retrieval of specific fraction magnitudes (cf. [64] for limited evidence of transfer effects in multiplication fact training).

\section{Differential neural activity patterns before training and possible implications}

Surprisingly, and not consistent with the previous literature on neural correlates of fraction processing brain activation before the training for the three tasks of interest revealed different activation patterns in the IPS associated with the numerical distance effect. For the non-symbolic line proportion comparison and the fraction-line proportion matching task we found significant neural activation patterns in the typical fronto-parietal network observed previously for proportion and fraction processing (cf. [40]). In particular, the line proportion comparison task led to increased activation in the right intraparietal sulcus, whereas the fraction-line matching task led to increased activation in the bilateral intraparietal sulcus. This is in line with research on brain activation for symbolic and non-symbolic magnitude processing $[70]$.

Interestingly, for the symbolic fraction magnitude comparison task activation in no cluster of voxels was modulated significantly by numerical distance before the training. This was surprising as previous studies on the neural correlates of fraction magnitude processing consistently reported IPS activity to be modulated by numerical distance for fraction magnitude processing. Importantly, the presented magnitudes did not differ between the three evaluation tasks. This means, that participants of our study were generally able to process the presented magnitudes. However, access to symbolic fraction magnitudes during the respective fraction comparison task might be reduced probably because of the bipartite nature of fractions [10]. Thus, fractions are more difficult to compare than for instance line proportions. 

reasons: i) Our fraction pairs did not involve fractions with common components. In this case, reasoning about the natural number components alone (i.e., processing numerators and denominators separately) might often not help to find the right solution when comparing two fractions without common components. However, we used fractions without common components as we wanted to specifically investigate and promote overall fraction magnitude processing. For instance, [71] found that mathematic experts showed a distance effect for overall fraction magnitude while comparing fractions without common components but not for components is typically susceptible to what has been called the natural number bias [72]. ii)

We used fractions with numerators and denominators ranging from 1 to 30 . This had two major reasons: a) the difficulty to match two item sets of fraction pairs on both overall numerical distance and problem size including only fractions without common components and b) the fact that we wanted to make sure that fractions were rather unfamiliar to participants to be able to investigate fraction learning on a neural level. Thus, participants may not have had a specific representation of the magnitude of the presented fractions prior to our study. In line with previous results of training studies, imaging results after the training showed that the processing of overall symbolic fraction magnitude was improved. In particular, distance related neural activation for symbolic fraction processing became significantly stronger from pre- to post-test in the bilateral intraparietal sulcus. This may indicate that our NLE training helped to establish easier access to the representation of overall fraction magnitude. These results are inconsistent with a NLE training study on natural numbers with pre-post-test fMRI comparing children with and without developmental dyscalculia [73]. After the NLE training both groups showed decreased activation of brain areas involved in number magnitude 
processing (for instance bilateral middle and superior frontal gyrus and left intraparietal sulcus). The authors interpreted these results as reflection of more automized processing of numerical magnitude after their training.

In this context, however, there is inconsistent evidence in the literature about the distance effect as an indicator for better numerical/ mathematical performance (see Moeller et al., 2011). On the one hand, there are studies showing that a larger distance effect was associated with poorer numerical/ mathematical performance $[74,75]$. On the other hand, there also are studies observing that a more pronounced distance effect was associated with better numerical/ mathematical performance [50,76]. To accommodate these inconsistent lines of evidence, [50] suggested that the relation between the size of the distance effect and mathematical performance might not be linear but curvilinear instead. In particular, these authors suggested that the size of the distance effect is depended on two factors: i) automated access to processed magnitudes decreases the distance effect whereas ii) increasing task complexity may increase the distance effect while processing magnitudes.

In line with this argument, it needs to be noted that we do not necessarily think that a larger distance effect indicates better number/ fraction magnitude processing. However, in the present study the increase of the distance effect on a neural level in symbolic fraction magnitude comparison might nevertheless indicate more automatic access to overall fraction magnitude as task complexity was very high. Moreover, due to the bipartite nature of fractions [10] overall fraction magnitude may not have been as automatically activated prior to the NLE training. Thus, after the NLE training our participants may have built a more coherent fraction magnitude representation reflected by a larger distance effect. In line with the hypothetical curvilinear model by [50] we think that the distance effect might decrease again after having established the magnitude representation with further training. 

line proportion comparison and fraction-line proportion matching task. This may indicate that improvement towards more automated activation on a neural level was not achieved through training as activation related to the numerical distance effect was already there before training. Again, this might reflect that the bipartite nature of fractions might have hindered automated magnitude processing of the fractions before training. In turn, non-symbolic proportions are further reflected by visual-spatial aspects which may have helped process the actual relative magnitude expressed as compared to symbolic fractions for which this relation needs to be built by participants themselves. Thus, the NLE training might not have changed processing of line proportions and fraction-line matching significantly on the neural level as the respective relative magnitudes may have already been processed before the training due to facilitation by visualspatial aspects of the presentation format. NLE based training of fractions aimed at improving conceptual knowledge of fraction magnitude. Importantly, the training did not only induce significant training effects on the bahavioral level but in particular also led to changes in brain activation associated with the processing of symbolic fraction magnitude. This indicates processes of neurofunctional plasticity in fraction learning. In the following, we will discuss implications of these results for education.

\section{Implications for education}

The final report of the National Mathematics Advisory Panel states that 'one key mechanism linking conceptual and procedural knowledge is the ability to represent fractions on a number line' (p. 28; [77]). However, fraction learning and understanding still is an educational challenge not only in the US but globally. The integrated theory of numerical development, postulates that one core basis of all (rational) numbers is their magnitudes and that these 
magnitudes can be represented on a mental number line [13]. Students difficulties with fractions often arise from missing conceptual understanding, which among other things but not exclusively involves an understanding of their magnitudes [78]. Therefore, it is a crucial step for students to learn that fractions are numbers with magnitudes that can be represented on a number line as well.

Accordingly, the recommendation to use number lines as an instructional tool to foster conceptual understanding of fractions is given in different guidelines for educational practice in fraction teaching (e.g., Teaching fractions [78] or Developing Effective Fraction Instruction for Kindergarten through $8^{\text {th }}$ grade [79]). This recommendation is supported by recent evidence from different intervention studies that used number lines as intervention tools and found significant improvements of children's performance and understanding of fractions [20-23,60]. With respect to educational practice, our results support the existing body of literature that processing of proportion and fraction magnitudes can be improved by NLE training. Moreover, to the best of our knowledge this is the first study indicating that such a training improves symbolic fraction processing as reflected by the numerical distance effect on a neural level. In particular, we argue that relative magnitude information of complex fractions may initially not be processed automatically within the IPS as indicated by the missing numerical distance related activation in the IPS before but significant activation associated with numerical distance after the training.

\section{Limitations}

When interpreting the results of the current study there are some limiting aspects that need to be considered. First of all, we are well aware that the current study is only a first step towards a better understanding of the underlying neural processes of fraction learning. In particular, this study investigated fraction learning on fractions more complex than those fractions first encounter in school. This was the case for two major reasons: i) Our fraction pairs 
did not consist of fractions with common components, which limits the number of available fractions when only considering those with numerators and denominators ranging from 1 to 9 . Therefore, we used fractions with numerators and denominators ranging from 1 to 30 allowing for proper matching of stimuli sets. ii) We ran our study with adult participants for whom we assumed that they should be more or less proficient with fractions with numerators and denominators up to 9. Thus, to be able to detect training effects we used more complex fractions. As such, to investigate fraction learning more fundamentally, less complex fractions (i.e., with single-digit numerators and denominators or even unit fractions) should be used. Moreover, our study investigated fraction processing in adult participants. To focus more on the fundamentals of fraction learning the developing brain should be investigated. A first attempt, to investigate developmental differences in fraction magnitude processing on a neural level is a study by [80]. In this study, the authors applied $2 \mathrm{~mA}$ bilateral tDCS and found that adults and children benefitted differently during fraction processing by tDCS applied to different areas of stimulation (IPS vs. dorsolateral prefrontal cortex (DLPFC)). However, imaging studies on the neural correlates of proportion and fraction magnitude processing in children are still missing.

\section{Conclusion}

It is well known that fractions are difficult to learn and understand not only for children and students but even adults and teachers $[7,81,82]$. Therefore, the ability to foster and improve fraction knowledge is of high educational importance. Apart from beneficial effects of NLE training on the behavioral level, our study provides first insights into the neural correlates of fraction learning. In particular, we did not observe numerical magnitude to significantly modulate brain activation before the training for the processing of symbolic fractions. This might indicate that overall fraction magnitude is not yet processed automatically before training. Thus, through the training participants might have built up more automated processing 
of overall fraction magnitude. As such, our results indicate a specific improvement of overall fraction magnitude processing through NLE training reflected on the neural level. This case of neuronal plasticity in fraction learning indicates neurofunctional changes elicited by training of educationally relevant content. Therefore, our study supports the importance of NLE trainings for fraction learning on a neurophysiological level.

References

[1] D.H. Bailey, M.K. Hoard, L. Nugent, D.C. Geary, Competence with fractions predicts gains in mathematics achievement, J. Exp. Child Psychol. 113 (2012) 447-455.

[2] R.S. Siegler, G.J. Duncan, P.E. Davis-Kean, K. Duckworth, A. Claessens, M. Engel, M.I. Susperreguy, M. Chen, Early predictors of high school mathematics achievement, Psychol. Sci. 23 (2012) 691-697.

[3] J. Torbeyns, M. Schneider, Z. Xin, R.S. Siegler, Bridging the gap: Fraction understanding is central to mathematics achievement in students from three different continents, Learn. Instr. 37 (2015) 5-13.

[4] M.J. Behr, I. Wachsmuth, T.R. Post, R. Lesh, Order and equivalence of rational numbers: A clinical teaching experiment, J. Res. Math. Educ. (1984) 323-341.

[5] K. Hart, Fractions, Math. Sch. 10 (1981) 13-15.

[6] R.S. Siegler, A.A. Pyke, Developmental and individual differences in understanding of fractions., Dev. Psychol. 49 (2013) 1994.

[7] X. Vamvakoussi, S. Vosniadou, Understanding the structure of the set of rational numbers: A conceptual change approach, Learn. Instr. 14 (2004) 453-467. 
[8] H. Lortie-Forgues, J. Tian, R.S. Siegler, Why is learning fraction and decimal arithmetic so difficult?, Dev. Rev. 38 (2015) 201-221.

[9] T.P. Carpenter, M.K. Corbitt, H.S. Kepner, M.M. Lindquist, R. Reys, Results of the second NAEP mathematics assessment: Secondary school, Math. Teach. 73 (1980) $329-338$.

[10] M. DeWolf, M.A. Grounds, M. Bassok, K.J. Holyoak, Magnitude comparison with different types of rational numbers., J. Exp. Psychol. Hum. Percept. Perform. 40 (2014) 71.

[11] R.S. Siegler, Magnitude knowledge: The common core of numerical development, Dev. Sci. 19 (2016) 341-361.

[12] R.S. Siegler, H. Lortie-Forgues, An integrative theory of numerical development, Child Dev. Perspect. 8 (2014) 144-150.

[13] R.S. Siegler, C.A. Thompson, M. Schneider, An integrated theory of whole number and fractions development, Cogn. Psychol. 62 (2011) 273-296.

[14] R. Gersten, R.F. Schumacher, N.C. Jordan, Life on the number line: Routes to understanding fraction magnitude for students with difficulties learning mathematics, J. Learn. Disabil. 50 (2017) 655-657.

[15] S.M. Göbel, S. Shaki, M.H. Fischer, The cultural number line: a review of cultural and linguistic influences on the development of number processing, J. Cross. Cult. Psychol. 42 (2011) 543-565.

[16] I. Berteletti, D. Lucangeli, M. Piazza, S. Dehaene, M. Zorzi, Numerical estimation in preschoolers., Dev. Psychol. 46 (2010) 545.

[17] D.C. Geary, M.K. Hoard, L. Nugent, J. Byrd-Craven, Development of number line 

representations in children with mathematical learning disability, Dev. Neuropsychol. 33 (2008) 277-299.

[18] R.S. Siegler, J.E. Opfer, The development of numerical estimation: Evidence for multiple representations of numerical quantity, Psychol. Sci. 14 (2003) 237-250.

[19] H.C. Barth, A.M. Paladino, The development of numerical estimation: Evidence against a representational shift, Dev. Sci. 14 (2011) 125-135.

[20] N. Hamdan, E.A. Gunderson, The number line is a critical spatial-numerical representation: Evidence from a fraction intervention., Dev. Psychol. 53 (2017) 587.

[21] C.A. Barbieri, J. Rodrigues, N. Dyson, N.C. Jordan, Improving fraction understanding in sixth graders with mathematics difficulties: Effects of a number line approach combined with cognitive learning strategies., J. Educ. Psychol. 112 (2020) 628.

[22] L.K. Fazio, C.A. Kennedy, R.S. Siegler, Improving children's knowledge of fraction magnitudes, PLoS One. 11 (2016) e0165243.

[23] K. Kiili, K. Moeller, M. Ninaus, Evaluating the effectiveness of a game-based rational number training-In-game metrics as learning indicators, Comput. Educ. 120 (2018) 1328.

[24] K. Kiili, K. Ojansuu, A. Lindstedt, M. Ninaus, Exploring the educational potential of a game-based math competition, Int. J. Game-Based Learn. 8 (2018) 14-28.

[25] J.L. Plass, U. Kaplan, Emotional design in digital media for learning, in: Emot. Technol. Des. Learn., Elsevier, 2016: pp. 131-161.

[26] K. Kiili, Digital game-based learning: Towards an experiential gaming model, Internet High. Educ. 8 (2005) 13-24.

[27] M. Csikszentmihalyi, Beyond boredom and anxiety: Experiencing flow in work and 
play, San Fr. (1975).

930

931

932

933

934

935

936

937

938

939

940

941

942

943

944

945

946

947

948

949

950

951

[28] K. Kiili, A. Lindstedt, M. Ninaus, Exploring characteristics of students' emotions, flow and motivation in a math game competition., in: GamiFIN, 2018: pp. 20-29.

[29] A. Landhäußer, J. Keller, Flow and its affective, cognitive, and performance-related consequences, in: Adv. Flow Res., Springer, 2012: pp. 65-85.

[30] D.C. Moos, E. Marroquin, Multimedia, hypermedia, and hypertext: Motivation considered and reconsidered, Comput. Human Behav. 26 (2010) 265-276.

[31] M. Csikszentmihalyi, Flow: The Psychology of Optimal Experience. Perennial Modern Classics, (1990).

[32] M. Ulrich, J. Keller, K. Hoenig, C. Waller, G. Grön, Neural correlates of experimentally induced flow experiences, Neuroimage. 86 (2014) 194-202.

[33] M. Ulrich, J. Keller, G. Grön, Neural signatures of experimentally induced flow experiences identified in a typical fMRI block design with BOLD imaging, Soc. Cogn. Affect. Neurosci. 11 (2016) 496-507.

[34] A. Perttula, K. Kiili, A. Lindstedt, P. Tuomi, Flow experience in game based learninga systematic literature review, Int. J. Serious Games. 4 (2017) 57-72.

[35] P. van Schaik, S. Martin, M. Vallance, Measuring flow experience in an immersive virtual environment for collaborative learningjcal_455 350.. 365, (2011).

[36] U. Konradt, R. Filip, S. Hoffmann, Flow experience and positive affect during hypermedia learning, Br. J. Educ. Technol. 34 (2003) 309-327.

[37] L.-A. Ho, T.-H. Kuo, How can one amplify the effect of e-learning? An examination of high-tech employees' computer attitude and flow experience, Comput. Human Behav. 26 (2010) 23-31. 
[38] L.A. Custodero, Observable indicators of flow experience: A developmental perspective on musical engagement in young children from infancy to school age, Music Educ. Res. 7 (2005) 185-209.

[39] R. MacDonald, C. Byrne, L. Carlton, Creativity and flow in musical composition: An empirical investigation, Psychol. Music. 34 (2006) 292-306.

[40] S.N. Jacob, A. Nieder, Tuning to non-symbolic proportions in the human frontoparietal cortex, Eur. J. Neurosci. 30 (2009) 1432-1442.

[41] J. Mock, S. Huber, J. Bloechle, J.F. Dietrich, J. Bahnmueller, J. Rennig, E. Klein, K. Moeller, Magnitude processing of symbolic and non-symbolic proportions: an fMRI study, Behav. Brain Funct. 14 (2018) 9.

[42] J. Mock, S. Huber, J. Bloechle, J. Bahnmueller, K. Moeller, E. Klein, Processing symbolic and non-symbolic proportions: domain-specific numerical and domaingeneral processes in intraparietal cortex, Brain Res. 1714 (2019) 133-146.

[43] J. Cui, L. Li, M. Li, R. Siegler, X. Zhou, Middle temporal cortex is involved in processing fractions, Neurosci. Lett. (2020) 134901.

[44] M. DeWolf, J.N. Chiang, M. Bassok, K.J. Holyoak, M.M. Monti, Neural representations of magnitude for natural and rational numbers, Neuroimage. 141 (2016) $304-312$.

[45] A. Ischebeck, M. Schocke, M. Delazer, The processing and representation of fractions within the brain: An fMRI investigation, Neuroimage. 47 (2009) 403-413.

[46] S.N. Jacob, A. Nieder, Notation-independent representation of fractions in the human parietal cortex, J. Neurosci. 29 (2009) 4652-4657.

[47] R.S. Moyer, T.K. Landauer, Time required for judgements of numerical inequality, 
Nature. 215 (1967) 1519-1520.

976

977

978

979

980

981

982

983

984

985

986

987

988

989

990

991

992

993

994

995

996

997

[48] M. Klabunde, M. Saggar, K.M. Hustyi, R.G. Kelley, A.L. Reiss, S.S. Hall, Examining the neural correlates of emergent equivalence relations in fragile $\mathrm{X}$ syndrome, Psychiatry Res. Neuroimaging. 233 (2015) 373-379.

[49] M. Piazza, P. Pinel, D. Le Bihan, S. Dehaene, A magnitude code common to numerosities and number symbols in human intraparietal cortex, Neuron. 53 (2007) 293-305.

[50] K. Moeller, S. Pixner, J. Zuber, L. Kaufmann, H.-C. Nuerk, Early place-value understanding as a precursor for later arithmetic performance-A longitudinal study on numerical development, Res. Dev. Disabil. 32 (2011) 1837-1851.

[51] F. Rheinberg, S. Engeser, R. Vollmeyer, Measuring components of flow: the FlowShort-Scale, Proc. 1st Int. Posit. Psychol. Summit. (2002).

[52] M. Ninaus, K. Kiili, J. McMullen, K. Moeller, Assessing fraction knowledge by a digital game, Comput. Human Behav. 70 (2017) 197-206.

[53] M. Ninaus, K. Moeller, J. McMullen, K. Kiili, Acceptance of Game-Based Learning and Intrinsic Motivation as Predictors for Learning Success and Flow Experience., (2017).

[54] \& M. Ninaus, Kiili, Wortha, Motivationsprofile bei Verwendung eines Lernspiels zur Messung des Bruchverständnisses in der Schule - Eine latente Profilanalyse., Psychol. Erziehung Und Unterricht. (n.d.).

[55] J.L. Booth, R.S. Siegler, Developmental and individual differences in pure numerical estimation., Dev. Psychol. 42 (2006) 189.

[56] D. Bates, M. Mächler, B. Bolker, S. Walker, Fitting linear mixed-effects models using 
lme4, ArXiv Prepr. ArXiv1406.5823. (2014).

999

1000

1001

1002

1003

1004

1005

1006

1007

1008

1009

1010

1011

1012

1013

1014

1015

1016

1017

1018

1019

1020

[57] A. Kuznetsova, P.B. Brockhoff, R.H.B. Christensen, lmerTest Package: Tests in Linear Mixed Effects Models. R package version 3.0-1, J Stat Softw. (2018).

[58] D. Makowski, The psycho package: an efficient and publishing-oriented workflow for psychological science, J. Open Source Softw. 3 (2018) 470.

[59] S.B. Eickhoff, K.E. Stephan, H. Mohlberg, C. Grefkes, G.R. Fink, K. Amunts, K. Zilles, A new SPM toolbox for combining probabilistic cytoarchitectonic maps and functional imaging data, Neuroimage. 25 (2005) 1325-1335. https://doi.org/10.1016/j.neuroimage.2004.12.034.

[60] E.A. Gunderson, N. Hamdan, L. Hildebrand, V. Bartek, Number line unidimensionality is a critical feature for promoting fraction magnitude concepts, J. Exp. Child Psychol. 187 (2019) 104657.

[61] R.M. Yerkes, J.D. Dodson, The relation of strength of stimulus to rapidity of habitformation, Punishm. Issues Exp. (1908) 27-41.

[62] S. Greipl, K. Moeller, M. Ninaus, Potential and limits of game-based learning, Int. J. Technol. Enhanc. Learn. (2020).

[63] A. Ischebeck, L. Zamarian, M. Schocke, M. Delazer, Flexible transfer of knowledge in mental arithmetic_-An fMRI study, Neuroimage. 44 (2009) 1103-1112.

[64] J. Bloechle, S. Huber, J. Bahnmueller, J. Rennig, K. Willmes, S. Cavdaroglu, K. Moeller, E. Klein, Fact learning in complex arithmetic - the role of the angular gyrus revisited, Hum. Brain Mapp. 37 (2016) 3061-3079.

[65] V. Menon, Memory and cognitive control circuits in mathematical cognition and learning, in: Prog. Brain Res., Elsevier, 2016: pp. 159-186. 
[66] E. Klein, K. Willmes, S.M. Bieck, J. Bloechle, K. Moeller, White matter neuroplasticity in mental arithmetic: changes in hippocampal connectivity following arithmetic drill training, Cortex. 114 (2019) 115-123.

[67] M. Amalric, S. Dehaene, Origins of the brain networks for advanced mathematics in expert mathematicians, Proc. Natl. Acad. Sci. 113 (2016) 4909-4917.

[68] M. Amalric, S. Dehaene, Cortical circuits for mathematical knowledge: evidence for a major subdivision within the brain's semantic networks, Philos. Trans. R. Soc. B Biol. Sci. 373 (2018) 20160515.

[69] M. Amalric, S. Dehaene, A distinct cortical network for mathematical knowledge in the human brain, Neuroimage. 189 (2019) 19-31.

[70] H.M. Sokolowski, W. Fias, A. Mousa, D. Ansari, Common and distinct brain regions in both parietal and frontal cortex support symbolic and nonsymbolic number processing in humans: A functional neuroimaging meta-analysis, Neuroimage. 146 (2017) 376-394.

[71] A. Obersteiner, W. Van Dooren, J. Van Hoof, L. Verschaffel, The natural number bias and magnitude representation in fraction comparison by expert mathematicians, Learn. Instr. 28 (2013) 64-72.

[72] Y. Ni, Y.-D. Zhou, Teaching and learning fraction and rational numbers: The origins and implications of whole number bias, Educ. Psychol. 40 (2005) 27-52.

[73] K. Kucian, U. Grond, S. Rotzer, B. Henzi, C. Schönmann, F. Plangger, M. Gälli, E. Martin, M. von ASTER, Mental number line training in children with developmental dyscalculia, Neuroimage. 57 (2011) 782-795.

[74] B. De Smedt, L. Verschaffel, P. Ghesquière, The predictive value of numerical magnitude comparison for individual differences in mathematics achievement, J. Exp. 

Child Psychol. 103 (2009) 469-479.

[75] I.D. Holloway, D. Ansari, Mapping numerical magnitudes onto symbols: The numerical distance effect and individual differences in children's mathematics achievement, J. Exp. Child Psychol. 103 (2009) 17-29.

[76] L. Rousselle, M.-P. Noël, Basic numerical skills in children with mathematics learning disabilities: A comparison of symbolic vs non-symbolic number magnitude processing, Cognition. 102 (2007) 361-395.

[77] NMAP, Foundations for success: The final report of the National Mathematics Advisory Panel, US Department of Education, 2008.

[78] L. Fazio, R.S. Siegler, Teaching fractions, International Academy of Education, 2011.

[79] R. Siegler, T. Carpenter, F. Fennell, D. Geary, J. Lewis, Y. Okamoto, L. Thompson, J. Wray, Developing Effective Fractions Instruction for Kindergarten through 8th Grade. IES Practice Guide. NCEE 2010-4039., What Work. Clear. (2010).

[80] S.M. Bieck, M. Splittgerber, K. Keil, R. Salvador, K. Moeller, V. Moliadze, P182 Bilateral tDCS indicates developmental differences in fraction magnitude processing, Clin. Neurophysiol. 131 (2020) e117.

[81] J.W. Stigler, K.B. Givvin, B.J. Thompson, What community college developmental mathematics students understand about mathematics, MathAMATYC Educ. 1 (2010) $4-16$.

[82] L. Ma, Knowing and Teaching Elementary Mathematics: Teacher's Understanding OfFundamental Mathematics in China and the United States, Lawrence Erlbaum Associates, Incorporated, 1999. 
1069 Stimuli and corresponding parameters (i.e, fraction magnitudes, numerical distance, problem size,

1070 matched distances and problem sizes within and between stimulus sets) for both item sets.

1071

\begin{tabular}{|c|c|c|c|c|c|c|c|}
\hline $\begin{array}{c}\text { fraction } \\
\text { pairs }\end{array}$ & $\begin{array}{l}\text { magnitude } \\
\text { fraction } 1\end{array}$ & $\begin{array}{l}\text { magnitude } \\
\text { fraction } 2\end{array}$ & $\begin{array}{c}\text { numerical } \\
\text { distance }\end{array}$ & $\begin{array}{l}\text { problem } \\
\text { size (PS) }\end{array}$ & $\begin{array}{c}\text { matched } \\
\text { distance } \\
\text { within and } \\
\text { between } \\
\text { sets } \\
\end{array}$ & $\begin{array}{c}\text { matched } \\
\text { PS within } \\
\text { and } \\
\text { between } \\
\text { sets }\end{array}$ & $\begin{array}{l}\text { Item } \\
\text { set }\end{array}$ \\
\hline$\frac{1}{8} \frac{5}{16}$ & 0,125 & 0,313 & 0,1875 & 0,438 & 0,022 & 0,078 & 1 \\
\hline$\frac{2}{3} \quad \frac{8}{11}$ & 0,667 & 0,727 & 0,061 & 1,394 & 0,022 & 0,078 & 1 \\
\hline$\frac{3}{8} \frac{11}{13}$ & 0,375 & 0,846 & 0,471 & 1,221 & 0,027 & 0,069 & 1 \\
\hline$\frac{1}{4} \frac{22}{25}$ & 0,25 & 0,88 & 0,63 & 1,13 & 0,027 & 0,069 & 1 \\
\hline$\frac{5}{12} \quad \frac{10}{17}$ & 0,417 & 0,588 & 0,172 & 1,005 & 0,006 & 0,066 & 1 \\
\hline$\frac{3}{14} \quad \frac{17}{25}$ & 0,214 & 0,68 & 0,466 & 0,894 & 0,006 & 0,066 & 1 \\
\hline$\frac{3}{19} \quad \frac{21}{29}$ & 0,158 & 0,724 & 0,566 & 0,882 & 0,006 & 0,066 & 1 \\
\hline$\frac{1}{24} \quad \frac{16}{25}$ & 0,042 & 0,64 & 0,598 & 0,682 & 0,006 & 0,066 & 1 \\
\hline$\frac{5}{27} \quad \frac{1}{26}$ & 0,185 & 0,039 & 0,147 & 0,224 & 0,008 & 0,045 & 1 \\
\hline$\frac{6}{23} \quad \frac{9}{25}$ & 0,261 & 0,36 & 0,099 & 0,621 & 0,008 & 0,045 & 1 \\
\hline
\end{tabular}




\begin{tabular}{|c|c|c|c|c|c|c|c|c|}
\hline$\frac{23}{27}$ & $\frac{13}{22}$ & 0,852 & 0,591 & 0,261 & 1,443 & 0,016 & 0,062 & 1 \\
\hline$\frac{19}{23}$ & $\frac{21}{25}$ & 0,826 & 0,84 & 0,014 & 1,666 & 0,016 & 0,062 & 1 \\
\hline$\frac{4}{7}$ & $\frac{5}{21}$ & 0,571 & 0,238 & 0,333 & 0,810 & 0,013 & 0,087 & 1 \\
\hline$\frac{3}{7}$ & $\frac{9}{22}$ & 0,429 & 0,409 & 0,020 & 0,838 & 0,013 & 0,087 & 1 \\
\hline$\frac{4}{5}$ & $\frac{17}{30}$ & 0,8 & 0,567 & 0,233 & 1,367 & 0,022 & 0,017 & 1 \\
\hline$\frac{5}{6}$ & $\frac{17}{22}$ & 0,833 & 0,773 & 0,061 & 1,606 & 0,022 & 0,017 & 1 \\
\hline$\frac{9}{14}$ & $\frac{15}{26}$ & 0,643 & 0,577 & 0,066 & 1,220 & 0,00017897 & 0,035 & 1 \\
\hline$\frac{9}{17}$ & $\frac{11}{29}$ & 0,529 & 0,379 & 0,150 & 0,909 & 0,00017897 & 0,035 & 1 \\
\hline$\frac{9}{10}$ & $\frac{14}{17}$ & 0,9 & 0,824 & 0,077 & 1,724 & 0,00017897 & 0,035 & 1 \\
\hline$\frac{9}{13}$ & $\frac{11}{17}$ & 0,692 & 0,647 & 0,045 & 1,339 & 0,00017897 & 0,035 & 1 \\
\hline$\frac{6}{13}$ & $\frac{7}{19}$ & 0,462 & 0,368 & 0,093 & 0,830 & 0,006 & 0,033 & 1 \\
\hline$\frac{6}{11}$ & $\frac{9}{26}$ & 0,545 & 0,346 & 0,199 & 0,892 & 0,006 & 0,033 & 1 \\
\hline 19 & 23 & & & & & & & \\
\hline 20 & 29 & 0,95 & 0,793 & 0,157 & 1,743 & 0,001 & 0,002 & 1 \\
\hline
\end{tabular}




\begin{tabular}{|c|c|c|c|c|c|c|c|c|}
\hline$\frac{19}{25}$ & $\frac{10}{11}$ & 0,76 & 0,909 & 0,149 & 1,669 & 0,001 & 0,002 & 1 \\
\hline & $\frac{6}{25}$ & 0,111 & 0,24 & 0,129 & 0,351 & 0,022 & 0,078 & 2 \\
\hline & $\frac{7}{10}$ & 0,625 & 0,7 & 0,075 & 1,325 & 0,022 & 0,078 & 2 \\
\hline$\frac{4}{9}$ & $\frac{25}{28}$ & 0,444 & 0,893 & 0,448 & 1,337 & 0,027 & 0,069 & 2 \\
\hline$\frac{2}{9}$ & $\frac{13}{14}$ & 0,222 & 0,929 & 0,706 & 1,151 & 0,027 & 0,069 & 2 \\
\hline$\frac{1}{18}$ & $\frac{25}{26}$ & 0,056 & 0,962 & 0,906 & 1,017 & 0,006 & 0,066 & 2 \\
\hline$\frac{1}{12}$ & $\frac{10}{29}$ & 0,083 & 0,345 & 0,262 & 0,428 & 0,006 & 0,066 & 2 \\
\hline$\frac{5}{19}$ & $\frac{17}{20}$ & 0,263 & 0,85 & 0,587 & 1,113 & 0,006 & 0,066 & 2 \\
\hline$\frac{9}{20}$ & $\frac{13}{27}$ & 0,45 & 0,482 & 0,032 & 0,932 & 0,006 & 0,066 & 2 \\
\hline$\frac{5}{23}$ & $\frac{7}{30}$ & 0,217 & 0,233 & 0,016 & 0,451 & 0,008 & 0,045 & 2 \\
\hline$\frac{7}{27}$ & $\frac{1}{22}$ & 0,259 & 0,046 & 0,214 & 0,305 & 0,008 & 0,045 & 2 \\
\hline$\frac{20}{27}$ & $\frac{11}{20}$ & 0,741 & 0,55 & 0,191 & 1,291 & 0,016 & 0,062 & 2 \\
\hline$\frac{19}{21}$ & $\frac{15}{19}$ & 0,905 & 0,790 & 0,115 & 1,694 & 0,016 & 0,062 & 2 \\
\hline
\end{tabular}




\begin{tabular}{|c|c|c|c|c|c|c|c|c|}
\hline$\frac{2}{5}$ & $\frac{3}{29}$ & 0,4 & 0,104 & 0,297 & 0,504 & 0,013 & 0,087 & 2 \\
\hline$\frac{1}{2}$ & $\frac{8}{17}$ & 0,5 & 0,471 & 0,029 & 0,971 & 0,013 & 0,087 & 2 \\
\hline$\frac{3}{4}$ & $\frac{13}{24}$ & 0,75 & 0,542 & 0,208 & 1,292 & 0,022 & 0,017 & 2 \\
\hline$\frac{8}{9}$ & $\frac{22}{29}$ & 0,889 & 0,759 & 0,130 & 1,648 & 0,022 & 0,017 & 2 \\
\hline$\frac{7}{13}$ & $\frac{11}{21}$ & 0,538 & 0,524 & 0,015 & 1,062 & 0,00017897 & 0,035 & 2 \\
\hline$\frac{9}{11}$ & $\frac{21}{26}$ & 0,818 & 0,808 & 0,011 & 1,626 & 0,00017897 & 0,035 & 2 \\
\hline$\frac{8}{15}$ & $\frac{13}{29}$ & 0,533 & 0,448 & 0,085 & 0,982 & 0,00017897 & 0,035 & 2 \\
\hline$\frac{9}{19}$ & $\frac{11}{25}$ & 0,474 & 0,44 & 0,034 & 0,914 & 0,00017897 & 0,035 & 2 \\
\hline$\frac{8}{13}$ & $\frac{9}{23}$ & 0,615 & 0,391 & 0,224 & 1,007 & 0,006 & 0,033 & 2 \\
\hline$\frac{6}{17}$ & $\frac{8}{27}$ & 0,353 & 0,296 & 0,057 & 0,649 & 0,006 & 0,033 & 2 \\
\hline$\frac{20}{23}$ & $\frac{12}{13}$ & 0,870 & 0,923 & 0,054 & 1,793 & 0,001 & 0,002 & 2 \\
\hline$\frac{15}{22}$ & $\frac{14}{15}$ & 0,682 & 0,933 & 0,252 & 1,615 & 0,001 & 0,002 & 2 \\
\hline
\end{tabular}

